\title{
Diffusion-weighted Imaging in Axial Spondyloarthritis: A Measure of Effusion or Does It Elicit Confusion?
}
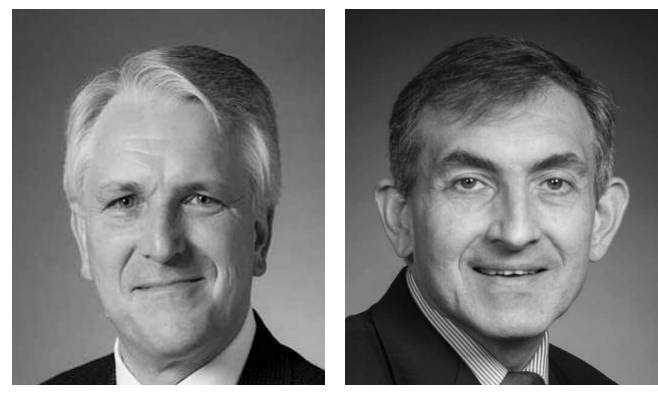

Clinical magnetic resonance imaging (MRI) is based on the resonance properties of hydrogen nuclei under the influence of fluctuating magnetic fields. The spinning proton's oscillations are enormously affected by its surrounding environment and so the MRI signal emitted from any tissue is profoundly altered by the proton concentration (very high in fat and water) and the tissue's chemical and physical composition. For example, hyaline cartilage has very high water content (appearing as bright as water on proton density imaging), but the collagen matrix profoundly affects the $\mathrm{T} 2$ properties of the protons, so that on T2-weighted imaging hyaline cartilage is dark. In conventional MRI, the tissues of interest are usually stationary and movement of any kind is most often just a darned nuisance.

In recent years, MRI is being used to investigate a whole variety of chemical and physical properties of tissues that was previously inconceivable. One such innovation is to measure the movement of free water within tissue at a microscopic level. Using diffusion-weighted imaging (DWI), it is possible for MRI to measure the speed and/or direction of movement of water molecules. The most common uses for DWI have been (1) in the imaging of space-occupying lesions to distinguish tumors from inflammatory masses; and (2) in the brain to provide additional information in ischemic events to detect cell necrosis (stroke) and clarify how much tissue is necrotic (nonviable) ${ }^{1}$. Most brain tissue has high water content and is highly vascularized. This differs from normal bone marrow, which has very high fat and very low water content and low perfusion, especially in adult fatty marrow. At the onset of brain ischemia, standard MRI sequences show no abnormality, but DWI very quickly shows decreased diffusion of water molecules, and in the appropriate setting, this is virtually pathognomonic for acute stroke. In distinguishing abscess from tumor, again the pathognomonic feature on DWI is decreased water diffusion in the abscess. The bottom line is that DWI in the brain is used to detect alterations in water molecule movement (increase or decrease) on a background of tissue where there is constant movement of water.
So what about DWI in bone marrow and arthritis imaging? A test that would provide greater ability to distinguish between inflammatory arthropathy and biomechanical bone marrow edema (BME) would be very useful. However, it is not clear that DWI will ever be superior to conventional approaches. DWI images are greyscale images that depict slow-moving water as bright, with a predetermined threshold for abnormal diffusion being selected by the operator - the $\mathrm{b}$ value $\left(\mathrm{s} / \mathrm{mm}^{2}\right)$. A higher $\mathrm{b}$ value indicates slower moving water. Apparent diffusion coefficient (ADC) maps illustrate the average speed of movement on a color scale. ADC maps and DWI images are derived from the same process, but do not show the exact same information. DWI images are essentially binary images with very high contrast showing slow-moving water as bright and everything else as dark. Seeing the bright lesion is easy - interpreting its significance is another matter. ADC maps are very colorful, but are hard to interpret and are primarily used to guide placement of regions of interest (ROI) for ADC calculation.

In this issue of The Journal, Bradbury, et al demonstrate that ADC values in the sacroiliac joint (SIJ) of patients with inflammatory sacroiliitis are increased compared to normal marrow - it is inconceivable that it would be otherwise ${ }^{2}$. DWI scans can provide numerical data that some researchers believe to be more objective than visual observation. While that may or may not be true, it is not unique to DWI and is also true of all MRI sequences. The strength of the signal being emitted from any ROI can be measured on any MRI sequence and compared to any other ROI on the same sequence. Sanal, et al measured contrast-to-noise ratios (CNR) for short-tau inversion recovery (STIR), contrast-enhanced fat-saturated T1, and DWI ${ }^{3}$. STIR had the highest CNR and appeared to be the most diagnostically useful. These measurements are not often done in clinical practice because the numerical information does not appear to be of sufficient additional value to merit the time and effort required to derive it. When an inflammatory reaction is more extensive or intense, the specificity of current MRI

See DWI in diagnosis of AS, page 771

Personal non-commercial use only. The Journal of Rheumatology Copyright (C) 2018. All rights reserved. 
sequences is very high. When the BME is subtle and the diagnostic challenge is greatest, DWI is unlikely to be useful because the lesions will be too small or too faint to allow accurate ADC calculation.

Bradbury, et al have expressed the opinion that DWI might provide both greater objectivity to the interpretation of MRI results and greater feasibility than scoring methods such as the Spondyloarthritis Research Consortium of Canada (SPARCC) MRI scores of the SIJ and spine ${ }^{2}$. Is this a reasonable conclusion of their methodology and findings? MRI signal strength, especially outside the brain, is highly variable and when using DWI to quantify the diffusivity of a lesion, various ROI have to be selected for both assessment of the lesion and determination of reference normal signal. First, an expert observer has to select an ROI in the SIJ on the STIR scan "within the lesion," in this case BME, to measure and then may have to select a second ROI "within a normal area" for comparison. How is this done? Bradbury, et al describe the process well: (1) nonblinded review of the scans was performed; (2) ADC maps were linked to the corresponding DWI and STIR images, to ensure accurate localization of the edema/ROI; (3) adjacent cortical bone, sclerosis, or fat metaplasia were excluded from the ROI to avoid volume averaging and erroneous ADC values; and (4) background reference signal along the midline of the sacrum at $\mathrm{S} 2$ level was also measured twice ${ }^{2}$. So you cannot create an accurate ADC value without interpreting T1, STIR, and DWI sequences first to allow accurate placement of the ROI. That means setting up scans to allow simultaneous assessment of T1W and STIR images and scrolling through consecutive slices to select optimal ROI for both the lesion and the reference normal signal. These decisions are entirely subjective based on the MRI interpreter's opinion of the findings on the MRI scan, and a second reader could select entirely different ROI. So is feasibility for clinical practice really superior to semiquantitative methods such as the SPARCC scores, where readers can record their observations on a simple Web-based scoring interface depicting a schematic of the SIJ (www.carearthritis.com)? The majority of patients with established axial spondyloarthritis (axSpA) have structural lesions in the SIJ, which could compromise selection of appropriate ROI. Further, when comparing MRI sequences, Boy, et al concluded that DWI did not significantly improve the diagnostic value of MR imaging for either experienced or less experienced radiologists and that DWI had far inferior sensitivity and no greater specificity than other sequences sensitive for BME/inflammation ${ }^{4}$. The demonstration of discrimination at a cross-sectional level between a small and selected group of patients with axSpA and nonspecific back pain controls and at a longitudinal level in patients with axSpA receiving tumor necrosis factor inhibitor therapy in this Australian study will require further validation in much larger and unselected cohorts of patients with back pain.
The entirely predictable observation that background reference signal in the sacrum varies among patients poses further challenges to the interpretation and potential utility of this quantitative imaging method. Sacral ADC values were significantly higher in patients with axSpA at pretreatment compared with patients who had nonspecific back pain, and significant variation was observed within the latter control group, likely related to age and sex and the associated differences in marrow fat. Consequently, corrected ADC values had to be derived by subtracting background signal in the sacral ROI from the score recorded by the ROI in the area of inflammation in the SIJ. This raises the possibility that discrimination for both diagnosis and treatment effect may vary according to sex, age, and other factors that may influence diffusion of water in healthy tissues.

The diffusivity of water molecules is dependent on multiple factors, and in bone marrow, many of these factors remain to be discovered. It should be emphasized that numerical data can be derived from any MRI sequence and objective quantification is not in any way special to DWI. Current processes for calculating ADC values in spondyloarthritis are highly subjective and require expert interpretation of conventional sequences, and at this point appear to offer no clear advantage over other widely accepted methods. The literature on this topic is confusing and high sensitivities and/or specificities for DWI/ADC mapping have usually been the result of careful selection of regions for analysis that are very obvious on conventional sequences.

\section{ROBERT G. LAMBERT, $F R C P(C)$}

Professor, Department of Radiology,

University of Alberta;

WALTER P. MAKSYMOWYCH, $\operatorname{FRCP}(C)$, Professor of Medicine,

University of Alberta,

Edmonton, Alberta, Canada.

Address correspondence to Dr. R.G. Lambert, Department of Radiology and Diagnostic Imaging, 2A2.41 WMC, University of Alberta, Edmonton, Alberta T6G 2B7, Canada. E-mail: rlambert@ualberta.ca

\section{REFERENCES}

1. de Carvalho Rangel C, Hygino Cruz LC Jr, Takayassu TC, Gasparetto EL, Domingues RC. Diffusion MR imaging in central nervous system. Magn Reson Imaging Clin N Am 2011;19:23-53.

2. Bradbury LA, Hollis KA, Gautier B, Shankaranarayana S, Robinson PC, Saad N, et al. Diffusion-weighted imaging is a sensitive and specific MRI sequence in the diagnosis of ankylosing spondylitis. J Rheumatol 2018;45:771-8.

3. Sanal HT, Yilmaz S, Kalyoncu U, Cinar M, Simsek I, Erdem H, et al. Value of DWI in visual assessment of activity of sacroiliitis in longstanding ankylosing spondylitis patients. Skeletal Radiol 2013;42:289-93.

4. Boy FN, Kayhan A, Karakas HM, Unlu-Ozkan F, Silte D, Aktas I. The role of multi-parametric MR imaging in the detection of early inflammatory sacroiliitis according to ASAS criteria. Eur J Radiol 2014;83:989-96

J Rheumatol 2018;45:729-30; doi:10.3899/jrheum.171479

Personal non-commercial use only. The Journal of Rheumatology Copyright $\odot$ 2018. All rights reserved. 